\title{
Shin-ichi Kuno \\ Division of linkage disequilibrium between absolute linkage disequilibrium and linkage equilibrium
}

Received: 7 March 2005/ Accepted: 19 April 2005/Published online: 4 June 2005

(C) The Japan Society of Human Genetics and Springer-Verlag 2005

\begin{abstract}
Linkage disequilibrium is the association between alleles in the allele distributions across linked loci and is intermediate in character between the dependence and the independence of allele distribution. This ambivalence makes linkage disequilibrium difficult to understand and to treat mathematically. To overcome this difficulty, an attempt was made to divide linkage disequilibrium between absolute linkage disequilibrium, which is a complete dependence of allele distribution, and linkage equilibrium, which is a complete independence. A matrix description of linkage disequilibrium showed that (1) linkage disequilibrium is divided between absolute linkage disequilibrium and linkage equilibrium, (2) a linkage disequilibrium state is characterized by the allele frequency in the first locus $p$, the relative content of absolute linkage disequilibrium $d$ and the linkage equilibrium variable $c$, and (3) $r$ is the geometric mean of both orientation's $d$. Division of linkage disequilibrium may make linkage disequilibrium straightforward to understand and to treat mathematically.
\end{abstract}

Keywords Linkage disequilibrium - Absolute linkage disequilibrium $\cdot$ Linkage equilibrium - Matrix ·

Division

Linkage disequilibrium is a most important key to understand the genomic structure in populations and is applied to the analyses to search for disease loci, e.g.,

S. Kuno

Clinical Genome Informatics Center,

Kobe University Graduate School of Medicine,

Kobe, Japan

Present address: S. Kuno ( $\square)$

Department of Clinical Study Management,

Translational Research Informatics Center,

Foundation for Biomedical Research

and Innovation, 1-5-4 Minatojima-minamimachi,

Chuo-ku, Kobe 650-0047, Japan

E-mail: omoikane@tri-kobe.org

Tel.: + 81-78-3032760

Fax: $+81-78-3032761$ indirect case-control analysis (Lange 2002) and transmission disequilibrium test (Spielman et al. 1993). Linkage disequilibrium is the association between alleles in the allele distributions across linked loci and is intermediate in character between the dependence and the independence of allele distribution. This ambivalence makes linkage disequilibrium difficult to understand and to treat mathematically. To overcome this difficulty, an attempt was made to divide linkage disequilibrium between absolute linkage disequilibrium, which is a complete dependence of allele distribution, and linkage equilibrium, which is a complete independence. A matrix description of linkage disequilibrium clearly realized this division.

In the present study, a linkage disequilibrium between two biallelic loci was investigated. The alleles and their relative frequencies were designated as described below: Two alleles of the first locus were $A$ with relative frequency $p$ and $a$ with relative frequency $1-p$, and those of the second locus were $B$ with relative frequency $q$ and $b$ with relative frequency $1-q$. Four haplotypes are allowed in this case, and the relative frequencies of the four haplotypes $A B, A b, a B$, and $a b$ were also designated $h_{A B}, h_{A b}, h_{a B}$, and $h_{a b}$, respectively. To determine the relation between the first and second loci, a matrix to array the relative frequencies of haplotypes with common alleles of the first locus in rows and those of the second locus in columns was constructed, as shown below:

\section{$\left(\begin{array}{ll}h_{A B} & h_{A b} \\ h_{a B} & h_{a b}\end{array}\right)$}

In this matrix, the relative frequencies of alleles of the first locus $(p, 1-p)^{T}$ and those of the second locus $(q, 1-q)^{T}$ are the horizontal and vertical marginal relative frequencies of haplotypes, respectively. In the matrix constructed as shown above, the linkage disequilibrium between the first locus and the second locus is determined by the linear transformation from the horizontal to the vertical marginal relative frequencies of the haplotypes. Designating $h_{A B} / p$ and $h_{a B} /(1-p)$ as $\alpha$ 
and $\beta$, respectively, a matrix to map the linear transformation from $(p, 1-p)^{T}$ to $(q, 1-q)^{T}$ is as shown below:

$\left(\begin{array}{cc}\alpha & \beta \\ 1-\alpha & 1-\beta\end{array}\right)$

As this matrix mapped the linear transformation and thus determines the linkage disequilibrium state, this matrix was named a "linkage disequilibrium matrix." In a linkage disequilibrium matrix, both $\alpha=1$ and $\beta=0$ or both $\alpha=0$ and $\beta=1$ signify absolute linkage disequilibrium. On the other hand, $\alpha=\beta$ signifies linkage equilibrium in a linkage disequilibrium matrix. Therefore, the division of the matrix mapping linkage disequilibrium into the matrices meeting both $\alpha=1$ and $\beta$ $=0$ or both $\alpha=0$ and $\beta=1$ or $\alpha=\beta$ should mean the division of linkage disequilibrium between absolute linkage disequilibrium and linkage equilibrium. In the case of $\alpha>\beta$, designating $\alpha-\beta$ and $\beta /(1+\beta-\alpha)$ as $d$ and $c$, respectively, the linkage disequilibrium matrix is developed, as shown below:

$$
\left(\begin{array}{cc}
\alpha & \beta \\
1-\alpha & 1-\beta
\end{array}\right)=d\left(\begin{array}{ll}
1 & 0 \\
0 & 1
\end{array}\right)+(1-d)\left(\begin{array}{cc}
c & c \\
1-c & 1-c
\end{array}\right)
$$

On the other hand, in the case of $\alpha<\beta$, linkage disequilibrium matrix was developed by designating $\beta-$ $\alpha$ and $\alpha /(1+\alpha-\beta)$ as $d$ and $c$, respectively, and the absolute linkage disequilibrium matrix with both $\alpha=0$ and $\beta=1$.

The equation shown above indicates that linkage disequilibrium is divided between absolute linkage disequilibrium and linkage equilibrium by a ratio of $d$ to $1-d$ where $d$ has already been used as a measure of linkage disequilibrium (Nei and Li 1980; Kaplan and Weir 1992; Devlin and Risch 1995). However, it was clear that $d$ signifies the relative content of absolute linkage disequilibrium in linkage disequilibrium in the present study. On the other hand, $c$ determines the state of linkage equilibrium. In linkage equilibrium, $h_{A B}: h_{A b}$ $=h_{a B}: h_{a b}$ is realized. The $c$ determines the ratio of $h_{A B}$ : $h_{A b}$.

A linkage disequilibrium state is determined by the three haplotypes' frequencies of the allowed four haplotypes. Each haplotype's frequency is limited by the other haplotypes' frequencies. In the matrix description of linkage disequilibrium, a linkage disequilibrium state is determined by the three variables, i.e., the allele frequency in the first locus $p$, the relative content of absolute linkage disequilibrium $d$, and the linkage equilibrium variable $c$. Both descriptions of linkage disequilibrium state consist of three variables. There is not a direct dependence between $d$ and $c$, but there is an indirect relation through $\alpha$ and $\beta$. However, $d$ and $c$ directly depend on $p$. Therefore, $d$ and $c$ depend on the order of loci, i.e., $d$ and $c$ calculated from the second locus is generally different from $d$ and $c$ calculated from the first locus, respectively. This antisymmetry of $d$ is a disadvantage for the measure of linkage disequilibrium. On the other hand, $r\left(\sqrt{\left(h_{A B} h_{a b}-h_{a B} h_{A b}\right)^{2} /(p(1-p) q(1-q))}\right)$ is the geometric mean of both orientation's $d$. Therefore, $r$ does not depend on the order of loci. If the symmetry of orientation of loci is needed, $r$ should be used as the geometric mean of both orientation's relative content of absolute linkage disequilibrium.

In the present study, it is shown that (1) linkage disequilibrium is divided between absolute linkage disequilibrium and linkage equilibrium, (2) a linkage disequilibrium state is characterized by the allele frequency in the first locus $p$, the relative content of absolute linkage disequilibrium $d$, and the linkage equilibrium variable $c$, and (3) $r$ is the geometric mean of both orientations' relative contents of absolute linkage disequilibrium $d$. This interpretation of linkage disequilibrium may make linkage disequilibrium straightforward to understand and to treat mathematically.

\section{References}

Devlin B, Risch N (1995) A comparison of linkage disequilibrium measures for fine-scale mapping. Genomics 29:311-322

Kaplan N, Weir BS (1992) Expected behavior of conditional linkage disequilibrium. Am J Hum Genet 51:333-343

Lange K (2002) Mathematical and statistical methods for genetic analysis, 2nd edn. Springer, Berlin Heidelberg, New York

Nei M, Li W-H (1980) Non-random association between electromorphs and inversion chromosomes in finite populations. Genet Res 35:65-83

Spielman RS, McGinnis RE, Ewens WJ (1993) Transmission test for linkage disequilibrium: the insulin gene region and insulindependent diabetes mellitus (IDDM). Am J Hum Genet 52:506-516 